\title{
Helical Interpolation Artifact
}

National Cancer Institute

\section{Source}

National Cancer Institute. Helical Interpolation Artifact. NCI Thesaurus. Code C87011.

A shape distortion in the z-axis resulting from the weighting function used in the helical interpolation algorithm. 